\title{
PILOTing towards a RAPID predictor of mortality for infectious pleural effusions
}

\author{
José M. Porcel (10) \\ Affiliation: Pleural Medicine Unit, Dept of Internal Medicine, Arnau de Vilanova University Hospital, IRBLleida, \\ University of Lleida, Lleida, Spain. \\ Correspondence: José M. Porcel, Pleural Medicine Unit, Dept of Internal Medicine, Arnau de Vilanova \\ University Hospital, Avda Alcalde Rovira Roure 80, 25198 Lleida, Spain. E-mail: jporcelpayahoo.es
}

@ERSpublications

The RAPID score can estimate short-term mortality in patients with pleural infections and should be considered the "gold standard" for outcomes assessment in this population https://bit.ly/31XctMK

Cite this article as: Porcel JM. PILOTing towards a RAPID predictor of mortality for infectious pleural effusions. Eur Respir J 2020; 56: 2002425 [https://doi.org/10.1183/13993003.02425-2020].

Depending on the imaging modality used, between 19\% (with chest radiographs) [1] and 54\% (with ultrasound) [2] of patients with community-acquired pneumonia have an accompanying pleural effusion. Most previous studies on parapneumonic effusions have focused on the necessity of fluid drainage $[3,4]$, with less attention being paid to the prognostic aspects. However, pleural infection (a term indistinctly used for parapneumonic effusions and empyemas) remains a serious condition associated with significant healthcare resource utilisation that portends a non-negligible mortality. In a large Danish registry of 6878 hospitalised patients with empyema the crude 30 -day mortality was about $10 \%$, though it ranged from $1.2 \%$ in those younger than 40 years to $20.2 \%$ in those aged $\geqslant 80$ years [5]. Supportively, in two large series of patients with pleural infections, the reported 30-day mortality rate was roughly $14 \%[6,7]$. Generally, figures for long-term prognosis are worse, with an observed 3-month and 1-year mortality of $23 \%$ and $42 \%$, respectively, in an Australian series of 561 adults with culture-positive pleural infections, two-thirds of which were hospital-acquired [8]. Moreover, a recent systematic review totalling 227898 patients with pleural infections found the median prevalence of pre-existing comorbidities (mainly respiratory and cardiac diseases) to be as high as $72 \%$, while the median length of hospital stay was 19 days [9].

In community-acquired pneumonia, two validated clinical prediction rules for prognosis are commonly used, namely the Pneumonia Severity Index (PSI) and CURB-65 (confusion, urea, respiratory rate, and age $\geqslant 65$ years). Both predict all-cause mortality at 30 days, but the former has higher discriminative power and is, therefore, recommended over the latter [10]. PSI assigns 10 points to the predictor variable "pleural effusion", since its independent association with mortality was demonstrated, and later replicated [11]. Can these scoring systems help to forecast a poor outcome in patients with parapneumonic effusions? The answer is elusive as hardly any publication addressing this question exists. In one retrospective study of 421 cases of complicated parapneumonic effusions PSI risk classes IV-V (i.e. >90 points) and CURB-65 $\geqslant 2$ points were identified as significant predictive factors for 30-day mortality (respective odds ratios of 4.7 and 5.5) [6]. Conversely, in a series of 4771 patients with pneumonia the electronic version of CURB-65 underestimated 30-day mortality when applied to the 690 who exhibited pleural effusions (7\% predicted versus $14 \%$ actual) [7].

In 2014, RAHMAN et al. [12] developed a prognostic model to specifically assist in predicting 3-month mortality in patients with pleural infections at the time of their presentation. The model, known as RAPID (Renal function, Age, Purulence, Infection source, Dietary factors), was derived using data from the MIST1 
clinical trial [13] and validated on the MIST2 cohort [14]. With the exception of non-purulent fluids, items that make up the RAPID model have a rational link with poor outcomes. Thus, it is expected that renal dysfunction, ageing, hospital-acquired infections and malnutrition negatively impact survival. RAPID scores 0 to 2 were classed as low risk, 3 to 4 as medium risk, and 5 to 7 as high risk of mortality at 3 months. The scoring system was accurate for prognosticating short-term mortality (area under the curve of 0.88 for the derivation cohort), but not surgical referrals. Subsequent to this pivotal research, a few retrospective studies have supported the RAPID score as a robust prognostic tool (table 1) [15-18], even for long-term mortality up to 5 years [15]. However, it can be argued that, to some extent, the RAPID model reflected the unique patient populations enrolled in the clinical trials from which it was generated, rather than the somewhat different features of real-world patients. In this sense, the study of CORCORAN et al. [19] in this issue of the European Respiratory Journal is uniquely relevant, in that it prospectively validates the performance characteristics of the RAPID scoring system in an observational cohort of 542 patients with pleural infections. Patients were recruited in four countries, and adherence to local protocols for pleural infection management was permitted, resulting in an investigation that faithfully reflected clinical practice. The study, under the acronym of PILOT (Pleural Infection Longitudinal Outcome Study), demonstrated that each 1-point increase on the RAPID scale was associated with an increase in 30-day mortality. The sum of this and previous studies on the subject, totalling 1453 patients with pleural infections, show that 3-month mortality rates are $11.8 \%$ overall and for those within RAPID low, medium and high-risk categories $1.9 \%, 11.7 \%$ and $35.6 \%$, respectively (table 1 ).

In the PILOT study, the authors used the concordance (C) statistic, a global measure of model discrimination, to assess the ability of the RAPID score to predict deaths. It was found that the $\mathrm{C}$ statistic for prediction of short-term mortality was 0.78 , thus indicating a good to strong predictive capacity $(0.5$ implying random concordance and 1 perfect concordance). $\mathrm{C}$ statistic is equivalent to the area under the receiver operating characteristic curve, but it has limitations, particularly for time-to-event data [20]. The $\mathrm{C}$ statistic is only a measure of discrimination, not calibration (i.e. how accurately the model's predictions match overall observed event rates), so it should be supplemented with other statistical and clinical measures. For instance, using both the positive predictive value and 1 minus negative predictive value can give information on what the patient's chances are of having an event (e.g. death), despite the model predicting they will or will not have one. Also, the Hosmer-Lemeshow statistic, though imperfect, is a means to assess model calibration.

Examining the possible outcomes of pleural infections not only gives patients an indication of what the future may hold, but can also theoretically help physicians make the right treatment decisions. Do patients in a RAPID high-risk category warrant more invasive initial therapy? Presumably, in patients with poor prognosis more efficient or invasive therapies, such as surgery, need to be discussed. However, the risk of death is not necessarily the same as the need for surgery. In fact, patients with a high RAPID risk score are not often good candidates for such procedures by virtue of their underlying fitness. On the other hand, conservative management, which includes prompt use of antibiotics [21] and intrapleural therapies, cures nearly $90 \%$ of the cases without rescue surgical interventions [22]. Whether RAPID score should be used to guide the care of patients with pleural infections needs to be specifically addressed in future clinical trials. Even if this prognostic data does not actually assist in the selection of appropriate therapy, it can help in counselling a concerned patient or relative about the expected future course of the illness. What

\begin{tabular}{|c|c|c|c|c|}
\hline \multirow[t]{2}{*}{ Study } & \multirow[t]{2}{*}{ Patients n } & \multicolumn{3}{|c|}{ Mortality at 3 months } \\
\hline & & RAPID score 0-2 & RAPID score 3-4 & RAPID score 5-7 \\
\hline \multicolumn{5}{|l|}{ RAHMAN et al. [12], 2014} \\
\hline Derivation cohort & 358 & $1 \%$ & $12 \%$ & $51 \%$ \\
\hline Validation cohort & 191 & $3 \%$ & $9 \%$ & $31 \%$ \\
\hline WHITE et al. [15], 2015 & 187 & $1.5 \%$ & $17.8 \%$ & $44.7 \%$ \\
\hline WoNG and YAP [16], 2016 & 77 & $2.9 \%$ & $27.6 \%$ & $28.6 \%$ \\
\hline TouRAY et al. [17], 2018 & 98 & $5.3 \%$ & $8.3 \%$ & $22.6 \%$ \\
\hline Corcoran et al. [19], 2020 & 542 & $2.3 \%$ & $9.2 \%$ & $29.3 \%$ \\
\hline TOTAL & $1453^{\#}$ & $1.9 \%$ & $11.7 \%$ & $35.6 \%$ \\
\hline
\end{tabular}

\footnotetext{
\#: of this total number of patients, 625,564 and 264 belonged to the low-, medium- and high-risk RAPID
} (Renal function, Age, Purulence, Infection source, Dietary factors) categories, respectively. 
can be substantiated so far is that the RAPID tool is simple, easily applicable at the bedside and accurate enough to be considered the "gold standard" for outcome assessment in patients with pleural infections.

Conflict of interest: J.M. Porcel has nothing to disclose.

\section{References}

1 Falguera $\mathrm{M}$, Carratalà $\mathrm{J}$, Bielsa $\mathrm{S}$, et al. Predictive factors, microbiology and outcome of patients with parapneumonic effusion. Eur Respir J 2011; 38: 1173-1179.

2 Reissig A, Copetti R, Mathis G, et al. Lung ultrasound in the diagnosis and follow-up of community-acquired pneumonia: a prospective, multicenter, diagnostic accuracy study. Chest 2012; 142: 965-972.

3 Porcel JM. Distinguishing complicated from uncomplicated parapneumonic effusions. Curr Opin Pulm Med 2015; 21: $346-351$.

4 Porcel JM, Valencia H, Bielsa S. Factors influencing pleural drainage in parapneumonic effusions. Rev Clin Esp 2016; 216: 361-366.

5 Søgaard M, Nielsen RB, Nørgaard M, et al. Incidence, length of stay, and prognosis of hospitalized patients with pleural empyema: a 15-year Danish nationwide cohort study. Chest 2014; 145: 189-192.

6 Park CK, Oh HJ, Choi HY, et al. Microbiological characteristics and predictive factors for mortality in pleural infection: a single-center cohort study in Korea. PLoS One 2016; 11: e0161280.

7 Dean NC, Griffith PP, Sorensen JS, et al. Pleural effusions at first ED encounter predict worse clinical outcomes in patients with pneumonia. Chest 2016; 149: 1509-1515.

8 Brims F, Popowicz N, Rosenstengel A, et al. Bacteriology and clinical outcomes of patients with culture-positive pleural infection in Western Australia: a 6-year analysis. Respirology 2019; 24: 171-178.

9 Cargill TN, Hassan M, Corcoran JP, et al. A systematic review of comorbidities and outcomes of adult patients with pleural infection. Eur Respir J 2019; 54: 1900541.

10 Metlay JP, Waterer GW, Long AC, et al. Diagnosis and treatment of adults with community-acquired pneumonia. An official clinical practice guideline of the American Thoracic Society and Infectious Diseases Society of America. Am J Respir Crit Care Med 2019; 200: e45-e67.

11 Zhang ZX, Yong Y, Tan WC, et al. Prognostic factors for mortality due to pneumonia among adults from different age groups in Singapore and mortality predictions based on PSI and CURB-65. Singapore Med J 2018; 59: 190-198.

12 Rahman NM, Kahan BC, Miller RF, et al. A clinical score (RAPID) to identify those at risk for poor outcome at presentation in patients with pleural infection. Chest 2014; 145: 848-855.

13 Maskell NA, Davies CW, Nunn AJ, et al. UK controlled trial of intrapleural streptokinase for pleural infection. $N$ Engl J Med 2005; 352: 865-874.

14 Rahman NM, Maskell NA, West A, et al. Intrapleural use of tissue plasminogen activator and DNase in pleural infection. N Engl J Med 2011; 365: 518-526.

15 White HD, Henry C, Stock EM, et al. Predicting long-term outcomes in pleural infections. RAPID score for risk stratification. Ann Am Thorac Soc 2015; 12: 1310-1316.

16 Wong D, Yap E. Pleural infection in a New Zealand centre: high incidence in Pacific people and RAPID score as a prognostic tool. Intern Med J 2016; 46: 703-709.

17 Touray S, Sood RN, Lindstrom D, et al. Risk stratification in patients with complicated parapneumonic effusions and empyema using the RAPID score. Lung 2018; 196: 623-629.

18 Yamazaki A, Ito A, Ishida T, et al. Polymicrobial etiology as a prognostic factor for empyema in addition to the renal, age, purulence, infection source, and dietary factors score. Respir Investig 2019; 57: 574-581.

19 Corcoran JP, Psallidas I, Gerry S, et al. Prospective validation of the RAPID clinical risk prediction score in adult patients with pleural infection: the PILOT study. Eur Respir J 2020; 56: 2000130.

20 Cook NR. Use and misuse of the receiver operating characteristic curve in risk prediction. Circulation 2007; 115: 928-935.

21 Porcel JM, Ferreiro L, Rami L, et al. Two vs. three weeks of treatment with amoxicilline-clavulanate for stabilized community-acquired complicated parapneumonic effusions. A preliminary non-inferiority, double-blind, randomized, controlled trial. Pleura Peritoneum 2020; 5: 20190027.

22 Porcel JM. Dual intracavitary therapy for pleural infections: leaving reluctance behind. Eur Respir J 2019; 54 : 1901001. 